\title{
Los Orígenes del Poblamiento Paleolítico en Europa: Una visión bioestratigráfica
}

\author{
José Yravedra Saínz de los Terreros *
}

\section{RESUMEN}

En este trabajo se revisan la representación faunistica existente en diferentes yacimientos del Paleolitico Inferior europeo, con el fin de poder dilucidar las posibles vias de paso que utilizaron los hominidos en el poblamiento inicial de Europa. De esta

manera la evidencia bioestratigráfica parece revelar una emigración de fauna africana a través de Gibraltar en el límite del Pliopleistoceno, pero todavia no hay ningún indicio claro que permita asegurar tal franqueamiento, ya que como se verá, esta posible fauna es algo discutible. Por otro lado los diferentes datos existentes permiten asegurar una ocupación de Europa en fechas anteriores al Pleistoceno Medio.

PALABRAS CLAVE Estrecho de Gibraltar, Bioestratigrafia, Pleistoceno Inferior, Pleistoceno Medio, Estudios Batimetricos.

\section{ABSTRACT}

In this paper the faunal representation of diferent European lower Paleolithic sites is discussed. The aim is to test the hypothesis that hominids might have crossed the Gibraltar straight in the earliest stages of the occupation of Europe. Biostratigraphic evidence reveals the passage of African faunas across the straight in the Plio-Pleistocene boundary. Howevert, there are insuficient dates to support their crossing the straight. Recent dates also support the presence of humans in Europe earlier than the Middle Pleistocene.

KEY WORDS

Gibraltar Strait, Biostratigraphy, Lower Pleistocene, Middle Pleistocene, Batimetric Studies.

1 Departamento de Prehistoria e Historia Antigua. UNED. Senda del Rey, s/n. 28040 Madrid. 


\section{INTRODUCCIÓN}

En este estudio se analizarán las posibles vías por las que tuvo lugar el poblamiento Paleolítico de Europa. Las cuales se relacionan con un paso desde Próximo Oriente hacia Europa Oriental y otro más directo pero más discutido, a través del Norte de África. Así, se revisan las faunas europeas del Pleistoceno Inferior y Medio, ya que según algunos autores ciertas especies del Sur de la Península Ibérica tienen un origen africano (Martínez Navarro, 1992 a, b; Iglesias et al, 1998). De esta forma se discuten estas evidencias paleontológicas y otros datos arqueológicos y batimétricos que pueden justificar un franqueamiento del Mediterráneo por Gibraltar o por el istmo sículo-tunecí.

En cualquier caso tanto el paso por el estrecho como por el Próximo Oriente es bastante complicado, ya que conlleva la superación de importantes barreras geográficas (Gibert, 1992; Gamble, 1995; Iglesias et al, 1998). Así, el Mar Mediterráneo sería un obstáculo insalvable entre el norte de África y el sur de Europa según Gamble (1995) y la mayor parte de la comunidad científica. En Próximo Oriente se encontrarían algunas depresiones montañosas del levante mediterráneo, como el Valle del Jordán, la Península del Sinaí, los montes Zagros y Tauro, que convirtieron a esta área en otra barrera de consideración (Gibert, 1992; Tchernov, 1992; Gamble, 1995) aunque siempre más asequible que su homónima marítima del norte de África.

Sobre esta cuestión numerosos autores han mostrado con evidencias claras el intercambio zoológico y cultural existente entre Europa, África y Asia en el cruce de caminos de Próximo Oriente. Así es demostrado que la procedencia de los équidos una vez llegados de Norte América a Asia, se extendieron desde aquí a África y Europa en una de las migraciones del Plioceno (Lindsay et al, 1980) ${ }^{2}$. Igualmente gran parte de las sucesiones faunísticas que tienen lugar durante los diferentes momentos al comienzo del Villafranquiense (2,5 m.a), y el límite Pliopleistoceno (1,6 m.a) son de procedencia asiática (Bonifay, 1979, 1980; Azzarolli, 1988; Turner, 1991; Martínez Navarro, 19922 a, b; Bosinski, 1996; Arribas \& Jorda, 1999) ${ }^{3}$. Hay muestras de varios yacimientos del Próximo Oriente

2 En este trabajo, estos autores describen como el antecesor de los équidos evolucionó en Norte América durante el Cenozoico, y como en el transcurso del Plioceno se extendió a Europa y África, atravesando el próximo oriente.

3 Hay que reconocer que la mayoria de estos autores y otros como Sesé (1994) y Sesé \& Sevilla (196) reconocen, que la aparición y desaparición de nuevas especies que tiene lugar en los diferentes momentos cronológicos del límite Mioceno-Pliceno, Plioceno-Pleistoceno y las distintas interfases pleistocenas suelen estar asociados a importantes cambios climáticos. 
con faunas de procedencia asiática y africana, que son claros indicativos del paso entre estos continentes. Por ejemplo Yiron (2,4 m.a), Ubeidiya, Bethelem y Dealul Mijlociu en Próximo Oriente y Rumania reflejan una simbiosis de faunas de procedencias diversas (Tchernov, 1981; Gibert, 1992; Bosinski, 1996; Iglesias et al 1998; Radulescu et al, 1998). También; Azzaroli et al (1988) reconoce la dispersión de fauna villafranquiense durante cuatro eventos diferentes, asociados a cambios climáticos. Así, el comienzo del Villafranquiense vendría dado por el «Evento del Leptobos" caracterizado por la llegada junto a esta especie, de grandes cérvidos y félidos, además de la desaparición de otras. El segundo evento es el de los équidos y el elefante que tiene lugar en el 2,5-2,6 m.a. A continuación sigue el «Evento del Lobo» en el límite Plio-pleistoceno (1,71,6 M.a) en el que llegaría Canis etruscus, Crocuta perrieri y Leptobos etruscus. Finalmente a este momento le sigue otro (1-0,9 m.a), en el que Leptobos desaparece frente a otros taxones nuevos como Allophaiomys y Canis arnensis. De esta forma se puede observar como según los trabajos de los diferentes autores parece claro el intercambio de faunas entre Europa y Asia a lo largo del Plioceno y el Pleistoceno (Azzaroli et al, 1988).

En lo concerniente a los primeros homínidos Turner (1991), Gibert (1992) y Bosinski (1996) han sugerido que la dispersión de estos suele estar asociado a los momentos de extinción y explosión de diferentes especies, así tendríamos el límite Mioceno-Plioceno para los primeros homínidos africanos y el del Pliopleistoceno para los primeros Homo. De la misma manera, la dispersión de estos primeros Homo suele ir ligada a la expansión de las sabanas (Turner, 1991; Gibert, 1992; Tchernov, 1992; Gabunia et al, 2000), lo que unido a lo comentado amteriormente sobre el intercambio faunístico de los tres continentes en el Próximo Oriente, puede indicar un poblamiento de Europa por vía terrestre desde esta región.

Frente a esta propuesta otros autores han defendido la posibilidad de la existencia de una ruta alternativa entre el norte de África y el sur de Europa, la cual se analizará a continuación (Alimen, 1975; Giles \& Santiago, 1987; Martínez Navarro, 1992 a, b; Iglesias et al 1998).

\section{EL PASO POR EL NORTE DE ÁFRICA}

El paso a Europa por el norte de África, es una cuestión que ha sido planteada durante bastante tiempo por diversos autores, los cuales coinciden en señalar que sólo hay tres tipos de datos que pueden ayu- 
darnos a resolver esta cuestión. El primero se refiere a las primeras acumulaciones líticas destacando principalmente su contenido lítico y su tipología. El segundo se centra en los análisis geológicos y batimétricos, que permite identificar el descenso de las profundidades marinas. Finalmente están los estudios bioestratigráficos cuya finalidad es apreciar posibles pasos y migraciones de fauna a través del estrecho.

\subsection{Los datos arqueológicos, batimétricos y bioestratigráficos de un paso por el Estrecho de Gibraltar}

En primer lugar haciendo mención a los estudios líticos de yacimientos antiguos, se podrían citar los trabajos de Biberson de los años 60, en los que comparando las industrias líticas del Paleolítico Inferior español con las del norte de África, se llegaba a la conclusión de que podían haber existido contactos entre ambos continentes (Biberson, 1961). También Pericot y Aguierre en la misma década plantearon la idea de un origen africano, para las industrias de la Península lbérica (Iglesias et al, 1998). En este trabajo de Iglesias et al (1998), se hace un recorrido historiográfico concerniente a este tema, mostrando como diversos autores se han preocupado por él, así Ripoll Perelló opinaba que pudo darse un paso de los estrechos aprovechando algún momento de aguas bajas (Iglesias et al, 1998; 31), también haciendo alusión a Querol y Santonja se dice que por las industrias del Aculadero el paso por Gibraltar debió realizarse en un momento temprano del Paleolítico Inferior, aunque Santonja es más partidario de una penetración por los Pirineos (Santonja, 1983). igualmente, Giles \& Santiago (1987) interpretan los complejos culturales pre-achelenses de la Península lbérica como una continuación de los del Magreb, ya que los triedros y hendedores son característicos del norte de África (Giles \& Santiago, 1987; 106).

En fin todas estas interpretaciones subrayan la idea de un posible paso amparándose en dos patrones; la similitud de las industrias norte africanas con las de la Península lbérica y las regresiones marinas que produjeron una disminución de los fondos marinos.

También en el ámbito europeo otros autores se han preocupado por esta cuestión, así Vaufrey en un trabajo de 1929, hace alusión a las migraciones concernientes al estrecho de Gibraltar y al istmo sículo-tunecí. En otro estudio, Alimen (1975) compara las industrias africanas con las del Paleolítico Inferior europeo (España, Francia y Sicilia), y llega a la conclusión de que es posible que exista algún contacto entre ambos continentes. 
Por otro lado Sahnouni (1998) ha mostrado como muchos de los yacimientos norte africanos son bastante problemáticos, por lo que adelantándome al transcurrir del texto, hay que decir que muchas de estas interpretaciones son problemáticas, en cualquier caso ya se analizará esta cuestión más adelante.

Igualmente en otros análisis y haciendo referencia a las investigaciones geológicas sobre la batimetría del Mediterráneo, algunos autores han llegado a resultados positivos. Así se pensó en un franqueamiento del estrecho aprovechando la sincronía de las regresiones marinas con las glaciaciones (Thoma, 1962). En otro tabajo tras analizar la batimetria actual, la sedimentación existente desde los tiempos achelenses, los movimientos tectónicos y las variaciones eustáticas, Alimen llega a unas conclusiones favorables en el franqueamiento del estrecho durante el transcurso del Riss, ya que aunque nunca dejo de haber agua entre los dos continentes, la distancia entre ambos debió ser bastante inferior a la actual (Alimen, 1975). Por lo que en el transcurso del Riss y el Mindel las regresiones marinas y otros condicionantes permitieron que en este tiempo la distancia entre los estrechos fuese inferior al disminuir la profundidad marina, ya que un descenso de los fondos marinos en torno $120 \mathrm{~m}$, pudo propiciar la existencia de una isla puente entre los dos continentes, dejando dos intervalos de $4 \mathrm{Km}$. Por otro lado el cruce de este canal parece más asequible que el istmo sículo-tunecí (Alimen, 1975) que nunca llegó a estar más próximo entre sí que en el Würm III, cuando llego a estar separado por $50 \mathrm{Km}$. Finalmente en otro trabajo Debenath et al (1986), amparándose en la idea de Alimen sugiere algún tipo de contacto entre las poblaciones aterienses del norte de África y las musterienses europeas, aunque como Alimen, también, se muestra escéptico ante un paso por Sicilia.

Por último, Zazo et al (1994) plantean una serie de cambios en el nivel del mar de los últimos 200000 años. Así para el estadio isotópico 7 b/a se detecta la presencia de dos oscilaciones en el nivel del mar, una en el $222 \mathrm{Ka}$ y otra en el $178 \mathrm{Ka}$, que pudo reducir el nivel marino. Por otro lado en el estadio isotópico 5 e datado en el $147 \mathrm{Ka}$ y el $128 \mathrm{Ka}$ se registra otra alteración del nivel marino, que a diferencia de todas las demás comentadas hasta ahora, registra un aumento del nivel en $5 \mathrm{~m}$.

Como ocurría con los estudios líticos, las conclusiones de los análisis batimétricos son también bastante problemáticos, ya que los diferentes trabajos se refieren a un marco temporal amplio y a diferentes periodos del Riss, el Würm e incluso del Mindel, que tiene como resultado un marco cronológico inconexo. Por otro lado al igual que los estudios líticos, la problemática de esto se comentará más adelante. 
Desde el punto de vista bioestratigráfico también son numerosos los diferentes trabajos que interpretan positivamente el paso por el estrecho, así volviendo al estudio de Iglesias et al (1998) queda manifiesta la presencia de determinadas especies africanas en el sur de la Península Ibérica, así en Cueva Victoria se ha identificado un cercopitécido conocido sólo en África, el Theropithecus sp (Gibert, 1992) ${ }^{4}$. En la cueva del Higuerón ha aparecido Gerbillus campestris con unos restos aislados y pendientes de confirmación ya que implica la existencia de un roedor africano en el Sudoeste español (Sesé \& Sevilla, 1996). En el yacimiento de Venta Micena se ha documentado un amplio conjunto faunístico de procedencia diversa. El cual adscrito al comienzo del Pleistoceno Inferior consta de varios taxones de procedencia diversa. (Martínez Navarro, 1992 a ${ }^{5}$ ). Entre ellos se puede apreciar como la procedencia de las distintas especies es de diferentes lugares, ya que se distingue una procedencia eurasiática, otra africana y una tercera autoctona (Gibert, 1992; Martínez Navarro, 1992 a, b; Arribas et al, 1996; Arrivas \& Jorda, 1999). Entre las de origen autóctono están Mammuthus meridionalis, Stephanorhinus etruscus; homotheriun latidens y Ursus etruscus. Entre las de procedencia asiática aparecen los rumiantes Bovini gen. et $s p$. Soergelia minor, Praeovibos sp, Capra alba, Caprini indet, Praemegaceros solihacus; Cervidae gen et sp; Equus cf altidens, Canis etruscus. Finalmente las especies provenientes de África son Canis falconeri, Pachycrocuta brevirostris, similar a la Hyaena bellax surafricana, Hippoppotamus anphibius antiqus, Homo sp., y el Meganteron whitei diferente del Meganteron cultridens de Eurasia y el Megantereon falconeri del subcontinente indio (Martínez Navarro, 1992 a; Arribas et al, 1996). En lo que respecta a otros yacimientos peninsulares ninguno muestra especies de origen africano, tan sólo la estación madrileña del Pontón de la Oliva datado al final del Pleistoceno Inferior refleja evidencias de Pachycrocuta brevirostris e Hippotamus amphibius antiqus (Sesé \& Soto, 2000).

En otro trabajo Turner (1991) observa que en las sucesivas migraciones que tiene lugar a lo largo de las diferentes etapas, al comienzo del Villafranquiense (2,5 m.a), el inicio del Pleistoceno Inferior (1,6 m.a) y la transición hacia el Pleistoceno Medio ( $0,9 \mathrm{~m} . \mathrm{a})$ esta caracterizada

\footnotetext{
4 Este yacimiento ha sido tradicionalmente atribuido al Pleistoceno inferior, pero como parece indicar las microfauna, quizás no se trate de un conjunto de dicho periodo, sino que se relacione más con un momento de transición entre el Pleistoceno Inferior-Medio. (Sesé \& Sevilla, 1996).

5 En este trabajo se exponen las diferentes listas faunísticas que se han ido desarrollando a lo largo de la investigación del yacimiento. Las especies expuestas corresponden a la lista definitiva, aceptada en 1990.
} 
principalmente por la procedencia asiática de nuevas especies, y africana de otras como el Mammuthus gromovi, el Megantereon cultridens y la Pachycrocuta perrieri para el comienzo del Villafranquiense $(2,5$ m.a), de Pachycrocuta brevirostris e Hippopotamus antiqus para el intervalo del 1,7-1,4 m.a, en el comienzo del Pleistoceno Inferior y de los carnivoros Panthera leo, Panthera pardus y Crocuta crocuta para la transición entre el Pleistoceno Inferior y Medio (0,9 m.a.). Por otro lado sus conclusiones se muestran algo dispares con el origen africano de las especies que definían Gibert (1992), Martínez Navarro (1992 a, b), Arribas et al. (1996) y Arrivas \& Jorda (1999), ya que para el límite del pliopleistoceno en el 1,7-1,4 $\mathrm{m}$. a. coinciden en reconocer la procedencia africana de Pachycrocuta brevirostris e Hippopotamus antiqus, pero difieren en el caso del Canis falconeri, que es de origen asiático según Turner y Azzaroli et al (1988), igualmente estos autores tampoco nombran a otros taxones de supuesto origen africano localizados sólo en el sur peninsular, como son Cercopitecus theropithecus y Meganteron whitei.

Por lo que resumiendo los trabajos de Turner (1991), Gibert (1992), Martínez Navarro (1992 a, b), Arribas et al. (1996) y Arrivas \& Jorda (1999) coinciden salvo alguna diferencia en ver un origen africano de las especies del limite pliopleistoceno (1,7-1,6 m.a) «Hippopotamus amphibius antiqus, Megantereon Withei, Canis falconeri, Theropithecus sp y Pachicrocuta brevirostris» y de las del 0,9 m.a «Panthera leo, Panthera pardus y Crocuta crocuta».

\subsection{Bioestratigrafía de los yacimientos más característicos del Pleistoceno Inferior Europeo}

Sí se analiza el registro faunístico de varias estaciones del Pleistoceno Inferior europeo se pueden observar como la supuesta procedencia africana de algunas de estas especies, es bastante discutible ya que muchas de los taxones identificados en Venta Micena aparecen también en yacimientos europeos de momentos contemporáneos o ligeramente anteriores. De la misma forma, aquellos taxones que Turner (1991) vincula con un proceder africano en el 0,9 m.a también han sido documentados en otros lugares europeos (Tabla 1).

En primer lugar se puede considerar como en los yacimientos cercanos a Venta Micena de Fuente Nueva III (1,6-1,5 m.a), Barranco León (1,5 m.a) y los cortijos de Doña Milagros y Alonso presentan Hippopotamus amphibius antiqus y Mamuthus meridionalis que coinciden con los taxones identificadas 
en Venta Micena (Gibert et al, 1992). Esto es significativo porque en el caso del Mamuthus meridionalis, algunos autores lo han considerado como una especie autóctona del sur de la Península lbérica (Gibert 1992; Martínez Navarro, 1992 a, b; Arribas et al, 1996; Arribas \& Jorda, 1999). Sin embargo, también ha aparecido en la estación argelina de Ain Hanech (1,9-1,7 m.a) (Sahnouni, 1998) lo que puede plantear ciertos problemas en el autoctonismo de este animal, ya que este lugar es más antiguo que los del Sur de la Península lbérica y además, también aparece en el asentamiento rumano de Dealul Mijlociu (1,7 m.a) (Radulescu et al, 1998) y Ubeydiya (1,4 m.a) (Tchernov, 1992), por lo que el autoctonismo de este taxón es bastante controvertido. Por otro lado la presencia de este taxón en el norte de África y el sur de Europa podría ir ligado a algún tipo de contacto entre ambos continenetes, pero las evidencias de Dealul Mijlociu y Ubeydiya también ofrecen otra posibilidad de paso por Próximo Oriente. Por otro lado de todas las especies documentadas en Ain Hanech sólo este taxón y el Hippopotamus amphibius antiqus aparecen en Venta Micena, por lo que de producirse un intercambio de fauna entre ambas regiones, este sería mayor.

Los otros taxones supuestamente autóctonos de Venta Micena como Stephanorhinus etruscus y Ursus etruscus (Martínez Navarro, $1992 \mathrm{a}, \mathrm{b}$ ), también han sido identificados en Dealul Mijlociu (Stephanorhinus etruscus y Ursus etruscus) y el Ursus etruscus en el yacimiento croata de Sandalja $(1,6$ M.a.) (Bosinski, 1996; 55), por lo que el autoctonismo de estos dos taxones como el del Mamuthus meridionalis puede responder a un origen más oriental.

Entre los supuestos taxones de procedencia africana documentados en Venta Micena se puede ver como algunos de ellos pueden tener un origen asiático. Así el caso del Canis falconeri tendría esta procedencia según Turner (1991), por otro lado esta especie ha sido identificada en Ubeidiya (Tchernov, 1992), lo que puede hacer pensar en un origen asiático frente a uno africano contradiciendo a Gibert (1992), Martínez Navarro (1992 a, b) Arribas et al (1996) y Arrivas \& Jorda (1999). En el caso de los restos de Pachycrocuta brevirostris también han aparecido en Dealul Mijlociu, por lo que se aproximan más a una llegada desde la región de Próximo Oriente que desde el norte de África. Además, Radulescu et al (1998) interpretan para este conjunto una procedencia anatólica y egea para muchas de las especies presentes. Finalmente en la identificación de Megantereon withei (Arribas et al, 1996), también parece presentar bastantes problemas ya que en las diferentes listas faunísticas que ha ofrecido el equipo de Venta Micena, ha tenido identificaciones distintas, así se lo ha llamado Megantereon sp, Megantereon cultridens adroveri y de nuevo en 1990 Megantereon sp, por lo que es bastante dudosa la identificación de Megantereon withei. (Tabla 1) 
Tabla 1. Taxones de procedencia africana según Turner (1991);

Gibert, (1992); Martínez Navarro, (1992 a, b); Arribas et al, (1996);

Arrivas \& Jorda, (1999), que aparecen documentados en el Pleistoceno Inferior-Medio europeo

\begin{tabular}{|c|c|}
\hline Yacimientos & Procedencia africana \\
\hline Venta Micena (1,6 m.a.) España & $\begin{array}{l}\text { Hippopotamus amphibius antiqus } \\
\text { Meganteron whitei } \\
\text { Canis falconeri } \\
\text { Pachycrocuta brevirostris } \\
\text { Mamuthus meridionalis }\end{array}$ \\
\hline Ain Hanech (1,9-1,7 m.a) Argelia & $\begin{array}{l}\text { Hippopotamus amphibius antiqus } \\
\text { Crocuta crocuta } \\
\text { Mamuthus meridionalis }\end{array}$ \\
\hline Cueva Victoria (Pleistoceno inferior-medio) España & Theropithecus sp \\
\hline Cueva del Higuerón (Pleistoceno Medio) España & Gerbillus campestris \\
\hline Pontón de la Oliva (Pleistoceno Inferior final) España & $\begin{array}{l}\text { Pachycrocuta brevirostris } \\
\text { Hippotamus amphibius antiqus }\end{array}$ \\
\hline Turner (1991) para el intervalo 1,7-1,4 m.a & $\begin{array}{l}\text { Pachycrocuta brevirostris } \\
\text { Hippopotamus antiqus }\end{array}$ \\
\hline Dealul Mijlociu (1,7 m.a.) Rumania & $\begin{array}{l}\text { Pachycrocuta brevirostris } \\
\text { Mamuthus meridionalis }\end{array}$ \\
\hline Vallonet (1,3 m.a) Francia & Panthera pardus, \\
\hline Fuente Nueva III (1,5-1,4 m.a.) España & $\begin{array}{l}\text { Hippotamos major } \\
\text { Mamuthus meridionalis }\end{array}$ \\
\hline Barranco león (1,6 m.a.) España & $\begin{array}{l}\text { Hippotamos major } \\
\text { Mamuthus meridionalis }\end{array}$ \\
\hline Ubeidiya (1,4-1,1 m.a.) Israel & $\begin{array}{l}\text { Canis falconeri } \\
\text { Crocuta crocuta, } \\
\text { Mamuthus meridionalis }\end{array}$ \\
\hline Isernia la Pineta $(0,9$ m.a.) Italia & $\begin{array}{l}\text { Panthera leo } \\
\text { Hippotamus amphibius }\end{array}$ \\
\hline Cullar Baza I (0,8 m.a.) España & $\begin{array}{l}\text { Crocuta crocuta, } \\
\text { Mamuthus meridionalis }\end{array}$ \\
\hline Dolina (0,78 m.a.) España & $\begin{array}{l}\text { Panthera leo } \\
\text { Crocuta crocuta }\end{array}$ \\
\hline Soleihac $(0,7$ m.a.) Francia & Hippotamus amphibius \\
\hline Tighenif (0,7 m.a.) Argelia & Crocuta crocuta \\
\hline $\begin{array}{l}\text { Treugolnaya (0,78-0,45 m.a.) Rumanía } \\
\text { Turner (1991) para la transición del Pleistoceno } \\
\text { Inferior al Medio }\end{array}$ & $\begin{array}{l}\text { Panthera leo } \\
\text { Panthera leo } \\
\text { Panthera pardus } \\
\text { Crocuta crocuta }\end{array}$ \\
\hline
\end{tabular}


Finalmente tan sólo los restos de Hippopotamus amphibius antiqus, Gerbillus campestris y Theropithecus $s p$ parecen tener una procedencia africana, claro esta que también muestran algún problema. De esta forma los restos del micromamífero de la cueva del Higuerón son muy aislados y todavia pendientes de confirmación según López Martínez (1972). En lo que respecta al cercopitécido de Cueva Victoria se ha visto como en el yacimiento pliopleistocénico de Nadah en la India han aparecido restos de Theropithecus sp (Singh, 1996). Por lo que en definitiva sólo los restos de hipopótamo son lo bastante sólidos como para pensar en un proceder africano, ya que las evidencias de hipopótamo del resto de los yacimientos sólo se han encontrado en fechas posteriores a los del entorno granadino salvo el caso argelino de Ain Hanech, por otra parte en los demás yacimientos en los que han aparecido hipopótamos, se refieren a subespecies diferentes que las de Venta Micena, así el Hippopotamos major, Hippopotamus gorgops, Hippopotamus belemoth sólo ha apercido en Dealul Mijlociu (Radulescu et al. 1988), Ubeydiya (Tchernow, 1992) y en Achalkalaki (Bosinski, 1996; 54), y en los que es Hippopotamus amphibius como es el caso de Isernia la Pineta (Anconetani et al., 1996) o Soleihac (Fosse \& Bonifay, 1991) corresponden a cronologías más modernas en plena transición con el Pleistoceno Medio.

Sí la fauna del Pleistoceno Inferior con un supuesto origen africano es bastante problemática, para la fauna del 0,9-0,7 m.a. que Turner adscribe a un proceder africano también es discutible. Así de las especies consideradas por este autor; Crocuta crocuta está documentada en el Pleistoceno inferior de Ubeidiya (Gibert, 1992; Tchernov, 1992) y en Ain Hanech (Sahnouni, 1998), al igual que en los yacimientos de la Gran Dolina (Aguirre, 1995), Cullar Baza I (Ruiz Bustos, 1984) y Tighenif (Sahnouni, 1998) de fechas más modernas. En el caso de Panthera pardus ha sido identificada en la Vallonnet (Echassoux, 1996) y, Panthera leo en el asentamiento italiano de Isernia la Pineta (Anconetani et al, 1996), el burgales de Gran Dolina (Aguirre, 1995), el rumano de Treugulnoya (Doronichev, 1996) lo que puede contradecir un origen africano, pues se encuentran en el transcurso del Pleistoceno Inferior en unos yacimientos más relacionados con una procedencia desde el Próximo Oriente que desde el norte de África.

Por to que en definitiva de todos los taxones comentados sólo el Hippopotamus amphibius puede haber llegado a Europa a través del estrecho de Gibraltar, ya que todos los demás, parecen estar más ligados a una migración desde Próximo Oriente que desde el norte de África. 


\section{POBLAMIENTO DE EUROPA}

En la exposición de los datos a los que me he ido refiriendo ha quedado manifiesta de forma indirecta una idea que hace alusión a la antigüedad del poblamiento paleolítico europeo en un momento anterior al Pleistoceno Medio, el cual para Turner $(1991,1995)$ fue muy favorable para los homínidos debido a la escasez de cánidos y hiénidos en el registro pleistocénico europeo, lo que ofrecía unas buenas posibilidades de carroñeo de aquellas carcasas producidas por félidos. Sin embargo, el registro paleofaunístico de los diferentes yacimientos europeos refleja un gran predominio de todo tipo de carnivoros, tanto cazadores como cazadores-carroñeros. Así entre los hiénidos hay Pachycrocuta perrieri, Pachycrocuta brevirostris, Pachycrocuta $s p$, Pachycrocuta vallonnetensis, Crocuta crocuta y Crocuta perrieri. Entre los cánidos también hay una amplia representación con Canis etruscus, Canis lupus mosbachensis, Canis arnensis, Canis falconeri, Cuon xenocyon $s p$, Cuon stehlini, Vulpes vulpes y Vulpes sp. Entre los osos aparece Ursus sp, Ursus etruscus, Ursus cuarctos mediterraneus y Ursus cf deningeri y, entre los félidos están el Homotherion crematidens; Megantereon megantereon; Megantereon cultridens, Panthera pardus, Panthera Gombaszogensis, Pantera leo fossilis, Lynx spelea, Felis sp, Felis leo, Felis gombaszogensis, Felis sylvestris, Felis caracal y Lynx issiodorensis (Fosse \& Bonifay, 1991; Gibert, 1992; Martínez Navarro, 1992 a, b; Tchernov, 1992; Aguirre, 1995; Darlas, 1995; Anconetanni et al, 1996; Bosinski, 1996; Ecchasoux, 1996; Doronichev, 1995; Radulescu et al, 1998; Gabunia et al., 2000)., por lo que la explicación que propone Turner para justificar un poblamiento de Europa en momentos anteriores al Pleistoceno Medio en función de la escasa presencia de carnívoros no parece muy cierta.

En cualquier caso las dataciones pliopleistocénicas de las industrias del entorno de Orce ${ }^{6}$, las asociaciones faunísticas que conllevan ${ }^{7}$ y las evidencias antrópicas existentes sobre las corticales óseas parecenn confirmar un poblamiento europeo anterior al Pleistoceno Inferior (Gibert et al., 1992 a; Gibert \& Jiménz, 1992; Jiménez \& Gibert, 1992).

También en Europa se han citado algunas estaciones de fechas tan antiguas como las Orce y a los yacimientos mencionados anteriormente de

6 Se refiere a los materiales de Venta Micena (1,6 m.a), Barranco del Paso (2-1,7 m.a), Barranco León 5 (1,9-1,8 m.a), Cortijo de Doña Milagros (1,6), Fuente Nueva III (1,4 m.a) y Cortijo de Don Alfonso (1,7 m.a) las cuales parecen estar bien contrastados (Gibert, 1992; Iglesias et al, 1998).

7 Barranco León 5, Venta Micena y Fuente Nueva están asociados a fauna (Gibert, 1992 a, b; Gibert \& Palmquost, 1992; Gibert et al, 1992 c; Martínez Navarro, 1995; Iglesias et al, 1992). 
Dealul Mijlociu, Sandalja, Vallonet, Ubeidiya, Yarimburgaz, Soleihac e Isernia la Pineta se podrían añadir los materiales de Dmanisi (Georgia) (Gabunia et al, 2000), Chillac (Francia), Rokossovo, Prezletize en la República Checa (Fridich, 1976), Korolevo en Ukrania (Gladiline \& Sitlivy 1991), Erq el Ahmar en Isrrael (Verosub \& Tchernov, 1991), Monte Pogiolo en Italia o Kärlich en Centro Europa (Bosinski, 1996).

Por otro lado ha quedado patente en numerosos estudios la problemática de algunos yacimientos, así son muy polémicas las evidencias antrópicas del famoso «hombre de Orce", pero también lo son las marcas de corte identificadas en este lugar, ya que están en unas proporciones muy bajas, lo que permite dudar de la veracidad de estas. En otros trabajos referentes al sur de la Península Ibérica, Montes Benárdez (1992) ha puesto de manifiesto la problemática de los yacimientos andaluces, criticando el hecho de que a pesar de la gran cantidad de estaciones localizadas, la gran mayoría de ellas carecen de excavaciones y estratigrafías adecuadas, concluyendo que "son pobres los datos referentes a los primeros pasos del hombre prehistórico en el sur peninsular" (Montes Bernárdez, $1992,7)$. En los estudios de la depresión de la Janda Fernández et al. (1987) ha observado que sus industrias se corresponden con unos momentos más modernos de lo que se venía reconociendo, por lo que debido a la problemática existente en torno a los yacimientos más antiguos de Europa, debemos esperar a nuevos datos de mayor fiabilidad. En cualquier caso lo que sí parece cierto es que Europa estuvo poblada en un momento anterior al Pleistoceno Medio tal y como indican los restos humanos y las dataciones de Atapuerca (Carbonell et al, 1999; Pares \& Pérez González, 1999). Y, por tanto, lo único que nos falta por saber es como se produjo el poblamiento y que ruta o rutas se siguieron.

Una de ellas es la del Próximo Oriente a la que ya me referí, la cual presenta un gran número de yacimientos con dataciones y materiales bien contrastados (Ubeydija, Dmanisi, Sandalja, Dealul Mjilociu etc.). La otra posible vía alternativa es la que circula en torno al franqueamiento por el estrecho de Gibraltar. Pero al contrario que en la primera los datos existentes son bastante problemáticos, no sólo por lo comentado en la Península lbérica sino que también por la situación existente en el Norte de África.

Asi; las industrias pre-achelenses existentes en Marruecos han sido puestas en entredicho, llegando a reconocer la inexistencia de una ocupación humana en el Marruecos Occidental Atlántico anterior al límite Bruhnes-Matuyama (Raynal et al, 1995), cuestionando los yacimientos de Rabat y Casablanca (Sidi Abderrahman, Tardiguer-er-Rahla y otros). Sólo 
Aïn Ainech en Argelia revela una datación más fiable gracias a su fauna villafranquiense (Sahnouni, 1998).

También los datos arqueológicos del Sur de la Península Ibérica son algo problemáticos. Así entre los datos faunísticos he observado una circunstancia similar ya que sólo el Hippopotamus amphibius, parece indicar algún contacto entre África y Europa, ya que las otras especies a las que hacen alusión los diferentes autores, han sido identificadas en otros yacimientos con cronologías similares a las del entorno de Orce en lugares próximos a Próximo Oriente. También los estudios batimétricos de Alimen (1975) y otros autores presentan problemas a la hora de pensar en una unión entre Europa y África, ya que nunca llegaron a juntarse ambos continentes ${ }^{8}$, siendo el mejor instante para atravesarlo en el Riss, cuando el Mediterráneo experimentó la mayor reducción de su profundidad, disminuiyendo la distancia entre los continentes a $11 \mathrm{Km}$ en el umbral situado entre Puente Paloma (al oeste de Tarifa) y Punta Malabata (al este de Tánger) ${ }^{9}$. Por lo que la situación ideal para establecer un contacto entre África y Europa seria en el transcurso del Pleistoceno Medio final ${ }^{10}$, situándolo en un momento mucho más moderno que el documentado para las primeras evidencias antrópicas de la Península lbérica.

\section{CONCLUSIÓN}

Como hemos visto en el desarrollo de este trabajo el poblamiento de Europa tuvo lugar en unas fechas bastante antiguas, sin embargo, no está tan claro saber que ruta escogieron para llegar. Es verdad que una ruta segura fue la del Próximo Oriente, pero es posible que se diera una segunda vía, en torno al cruce del estrecho de Gibraltar, ya que el cruce por el istmo sículo-tunecí no parece muy viable, dados los $50 \mathrm{Km}$. que debieron separar ambas costas en el momento más favorable.

Respecto al franqueamiento por Gibraltar, la evidencia arqueológica y faunistica no contradice esta posibilidad, aunque como he indicado la interpretación de algunos datos no esta exenta de problemas. En primer

8 Tan Sólo la crisis salina producida por la evaporación y la falta de comunicación con el Atlántico en el Mesiniense hace 5 millones de años ocasiono que se uniera el norte de África con el sur de la Península Ibérica.

9 Esta reducción sería tal, si suponemos un descenso de $120 \mathrm{~m}$ en el nivel marino.

10 No hay que olvidar que durante el Pleistoceno Medio los homínidos sí tenian cierta capacidad de franquear pequeños istmos, como ha quedado patente en la documentación de yacimientos del Paleolítico inferior en Sicilia, un lugar que a lo largo del Pleistoceno nunca perdió su insularidad. 
lugar se ha visto como el paso de determinadas especies por el estrecho es discutible y sólo el hipopótamo parece tener una procedencia africana clara, lo cual tampoco es de extrañar sí pensamos en las extraordinarias condiciones natatorias de este animal. De la misma manera los datos batimétricos indican que en los últimos 2 millones de años nunca se mantuvo unida Europa y África por un puente de tierra, e incluso en el momento en el que más se redujo el nivel del agua en torno a los $120 \mathrm{~m}$, durante el Riss no es suficiente para suponer una gran reducción de la distancia entre los dos continentes.

Por otro lado sí damos por buena una posible emigración africana en el Pleistoceno Inferior a través el estrecho de Gibraltar, habría que preguntarse por que en el resto del Pleistoceno y sobre todo en el transcurso del Pleistoceno Superior no se siguió con este tipo de contactos. Del mismo modo sería paradójico el hecho, que los neandertales quedaran confinados a Europa extendiéndose sólo en dirección hacia Próximo Oriente, de la misma forma que la expansión de los humanos modernos sólo aprovechara la vía de Próximo Oriente, sin detenerse en la otra posibilidad que supondría pasar por el Norte de África.

Numerosos autores se han referido siempre a la Península Ibérica como un refugio para muchas especies, no sólo por las peculiaridades climáticas que supone la Península respecto al resto de Europa (Sesé, 1994; Sesé \& Sevilla, 1996), sino que también por la barrera geográfica que supuso el mar (Currant, 1994). Por lo que en función de lo dicho en este trabajo no es posible defender un cruce del estrecho de Gibraltar durante el pleistoceno Inferior, ya que los datos batimétricos indican que durante el Pleistoceno Europa y el norte de África nunca estuvieron unidos, los paralelos líticos son problemáticos y la fauna parece estar más relacionada con una procedencia desde Próximo Oriente.

\section{BIBLIOGRAFÍA}

AguirRe, E. (1995): “Registro faunístico Pleistoceno antiguo en Atapuerca (Burgos)». Trabajo de Prehistoria 52. 47-60.

Alimen, N.H. (1975): “Les isthmes hispanoma-roquian et siculo-tunisien aux temps acheuléens". L'Antropolgie 79. (3). 399-436.

Anconetani P.; Díez, C. \& Rosell J. (1996): Intentional bone fracturing for marrow extraction in Atapuerca and Isernia La Pineta (Italy) Lower Paleolithic sites. En XIII International Congress of Prehistoric and protohistoric sciencies Forlì. Italia 8/14 Sept. 1996. Procedings.(ed. Facchini, F.; Palma di Cesnola, A.; Piperno, M. \& Pereto, C): 445-453.

arribas Herreta, A.; Palmouist, P. \& Martínez Navarro, B. (1996): «Estudio tafonómico cuantitativo de la asociación de macromamíferos de Venta Micena". Comunicaciones de la II Reunión de Tafonomia y Fosilización. Madrid: 27-38. 
Arribas Herrera, A. \& Jorda, J. F (1999): Los macromamiferos del Cuaternario kárstico de Guadalajara (Castilla la Mancha). En Aguirre, E. \& Rábano, I. (ed.) La huella del Pasado. Fósiles de Castilla la Mancha. Patrimonio Arqueológico Histórico Castilla la Mancha: 329-349.

Azaroli, A. Giuli C. De; Ficarelli G. \& Torre D. (1988): "Late Pliocene to early midle Pleistocene. Mammals in Eurasia faunal sucession and dispersal events". Palaeogeography, Palaeoclimatology, Palaeocology 66: 77-100.

Biberson, P. (1961): "Le cadre paléogeographique de la Préhistoire du Maroc" Atlantique. Publications du Service des Antiquites du Maroc. Fasc. 16. Rabat.

BONIFAY, M.F. (1979): “Interrelations entre les grandes faunes plio-pléistocènes euroasiatiques et origine des faunes françaises". Quaternaria 21: 9-16.

- (1980). «Relations entre les données isotopiques océaniques et l'histoire des grandes faunes européennes Plio-Pléistocènes". Quaternary Research. 14.: 251-262.

BosINSKI, G. (1996): Les origense de l'homme en europe et en Eurasia. Edit.Errance.

Carbonell, E.; Bermudez de Castro, J. M.A; Arsuaga, J.l.; Diez, J. C., Rosas, A.; Cuenca, G.; Bescois, R; Sola, R.; Mosouera, M; Rodríguez, X.P. (1995): "Lower Pleistocene Hominity and artefacts from Atapuerca-TD6 (Spain)". Sciencie. 269: 826-828.

CurRant, A. (1994): "Quaternary Manmals of Gibraltar". En Gibraltar during the Quaternary. AEQUA Monografics, 2.: 115-117.

DARLAS. A. (1995): “The earliest ocupation of Europa: The Balkans». En Roebroecks, W \& Van Kolfschoten, $T$ (1995) The earliest ocupation of Europe. University of Leiden.: 51-59.

Debenach, A.; Raynal, J. P.; Roche, J; Texier, J. P. \& Ferembach, D. (1986): «Stratigraphie, habitay, typologie et Devenir de l'Aterien Marocain. Donnéens Récents". L'Antropologie 90,.(2): 233-246.

DoronicheV V.B. (1996): "Treugoinaya cave a New Lower Paleolithic site on the Northern Caucasus". En XIII International Congress of Prehistoric and protohistoric sciencies Forli. Italia 8/14 Sept. 1996. Procedings.(ed. Facchini, F.; Palma di Cesnola, A.; Piperno, M. \& Pereto, C): 387-392.

Echassoux, A. (1996): "Les grandes mamiferes de la Grotte du Vallonet. (Roquerbrune-CopMartin), Alpes maritimes. Francia. Constitution de l'assemblage". En XIII International Congress of Prehistory and Protohistory Sciencie. Forli Italia 8-14 sepetiembre Procedings. Arias ed: $439-446$.

Fernandez llebrez B.; Mateos Alonso V. \& Ramírez Delgado, J. R. (1987): “Los yacimientos paleolíticos de la depresión de la Janda (Provincia de Cádiz)». Congreso Internacional del Estrecho de Gibraltar. Actas Tomo 1: 87-96.

Fosse, P. \& BonIFAY, M. F. (1991): "Les vestigies osseaux de Soleilhac, Approache Taphonomique» Bonifay, E. \& Vandermersch B. (1991). Les premiers Européens, Editions du comité dels travaux historiques et scientifiques actes du 114 Congreés National des Societe savantes. 1989: 115-133.

FRIDRICH, J. (1976): "The first industries from eastern and South-eastern central Europe». Les premieres industries de l'Europe, K. Valloch (edit), UISPP, congrés IX, Coll VIII Niza: 8-23.

GabUNIA, L.; VEKUA, A. \& LoRdKIPANIDZE, D. (2000): "The environmental contexts of early human occupation of Georgia (Transcaucasia)". Journal of Human Evolution. Mayo: 785-802.

GamBLE, C. S. (1995) "The earliest occupation of Europe: the envioronmental background". En Roebroecks, W \& Van Kolfschoten, T (1995) The earliest Occupation of Europe. University of Leiden: 280-295.

GiBeRT, J. (1992): “Evolución y dispersión de los homínidos desde una perspectiva ecológica». En Gibert, J (1992). Presencia humana en el Pleistoceno Inferior de Granada y Murcia. Proyecto Orce-Cueva Victoria (1988-1992): 445-458.

GIBERT, J. \& PALmquist, P. (1992): "Aplicación de análisis del frontal de fragmento craneal de Homo sp en Venta Micena". En GiBert, J (1992). Presencia humana en el Pleistoceno Inferior de Granada y Murcia. Proyecto Orce-Cueva Victoria (1988-1992): 371-390.

GIBERT, J. \& JIMÉNEZ C. (1992): "Estudio preliminar de las marcas de acción antrópica en el yacimiento de Venta Micena". Congreso Nacional de Arqueología: 453-463.

Gibert, C. K.; Fernández Caro; Martinez, B. \& Caparocci, R. (1992 a): «Roturas antrópicas en las Cuevas de Venta Micena". Congreso Nacional de Arqueología.: 431-444.

GiberT, J.; IGLesiAs, A.; Maillo, A. \& Gibert, L. (1992 b): “Industrias líticas en el Pleistoceno inferior en la región de Orce». En GlBeRT, J. (1992). Presencia humana en el Pleistoceno Inferior de Granada y Murcia. Proyecto Orce-Cueva Victoria (1988-1992).: 219-283. 
Giles Pacheco, F. \& Santiago Peret, A. (1987): “El poblamiento paleolítico del sur de la Peninsula Ibérica en el Pleistoceno Inferior a través de Gibraltar". Congreso Internacional del Estrecho de Gibraltar. Actas.: 97-109.

Iglesias DiÉGuez, A., Gibert Cols, J., Gibert Beotas, L. (1998): «La penetración de los homínidos por el Estrecho de Gibraltar en el contexto general de su dispersión». Gallaecia 17: 29-46.

JIMÉNEZ, C. \& GiBERT, J. (1992): “Estudio comparativo de las marcas de corte de Venta Micena". En Gibert, J (1992). Presencia humana en el Pleistoceno Inferior de Granada y Murcia. Proyecto Orce-Cueva Victoria (1988-1992): 307-339.

LindSAY, E. H.; OPDYKE, N. D.\& JOHNSON, N. M. (1980): «Pliocene dispersal of the horse Equus and late Cenozoic mammalian dispersal events" Nature 287-11.9.:135-138.

López MaRTínez, N. (1972): “Los micromamíferos del Cuaternario de Rincón de la Victoria». Boletín de la Real Sociedad española de Historia Natural (Geología), 70: 223-233.

Martinez Navarro B. (1992 a) "Revisión sistemática de la fauna de macromamíferos del Yacimiento de Venta Micena (Orce, Granada, España)». En Gibert, J. (ed) Proyecto OrceCueva Victoria. Presencia Humana en el Pleistoceno Inferior de Granada y Murcia: 21-53.

Martinez NavarRo, B. (1992 b): «Estudio cuantitativo y consideraciones paleobiológicas de la Comunidad de Mamíferos del Yacimiento de Venta Micena». EN GIBERT, J. (1992). Presencia humana en el Pleistoceno Inferior de Granada y Murcia. Proyecto Orce-Cueva Victoria (1988-1992): 155-188.

MONTES BernardeEz, R. (1992): “Los primeros grupos humanos depredadores en el Sur de la Península (Andalucia, Murcia y Albacete)" Munibe 43: 3-12.

Pares, J. M. ${ }^{a}$ \& Pérez Gonzalez, A. (1995): «Paleomagnetic age for hominid fossils at Atapuerca archaeological site, Spain». Sciencie 269: 828-829.

Radulescu, C.; Samson, P. M. \& Stinca, E. (1998): "Cadre bioestratigraphique du Paleolithique Inférieur en Roumanie». Quaternaire 9: 283-290.

Raynal J. P; Magoga, L.; SbHi-Alaoui, F-Z. \& Gerads; D. (1995): «The earliest occupation of Atlantic Morocco: The Casablanca evidence". En RoEBROECKS, W. \& VAN KolfSCHOTEN, T (1995) The earliest ocupation of Europe. University of Leiden: 255-261.

Ruiz Bustos, A. (1984): “El yacimiento paleontológico de Cúllar de Baza I.» Investigación y Ciencia 91: 20-29.

SAHNOUNI, H. (1998): The Lower Palaeolithic of the Magherb. Excavation and analyses at Ain Hainech, Algeria. BAR International Series 689. Oxford.

SANTONJA, M. (1983): "Indicios arcaicos de la presencia humana en el Interior de la Península Ibérica". Revista de Arqueología, 29: 24-28.

SESE, C. (1994): "Paleoclimatological interpretation of the Quaternary small manmals of Spain". Geobios. 27.6.94: 753-717.

Sesé, C \& SEVILLA, P. (1996): «Los macromamíferos del cuaternario peninsular español. Cronoestratigrafía e implicaciones bioestratigráficas". Revista española de Paleontología. Número extraordinario. Junio: 278-287.

SesE, C. \& Soto, E. (2000): "Vertebrados del Pleistoceno de Madrid». En J. Morales (Coord) Patrimonio Paleontológico de la Comunidad de Madrid. Consejería de Educación de la Comunidad de Madrid. 216-243.

SINGH, M.P (1996): "First Record of the Middle Pleistocene Hominid and his Culturas from the Pinjor Formation of the Indian Subcontinent". En XIII International Congress of Prehistoric and protohistoric sciencies Forli. Italia 8/14 Sept. 1996. Procedings: 13-28.

TCHERNOV, E. (1981): "The bioestratigraphy of the Middle East». En Cauvin, J. \& Sanlaville. The Préhistoire du Levant: Chronologie et organisation de l'espace depuis les originies jusqu'au VI e millénaire. Paris CNRS: 67-97.

- (1992): «Biochronology, Paleoecology and dispersal events of hominids in the Southern Levant". En (AKazAwa, T.; AOKI, K.\& KIMURA, T).The evolution and dispersal of modern humans in Asia: $149-188$.

Thома, A. (1962): "Le deploiement évolutif de L'Homo Sapiens". Antropologica Hungárica n. ${ }^{\circ}$ 1-2. Budapest.

TURNER, A. (1995): "Variaciones regionales en la fauna de grandes mamíferos del Pleistoceno inferior y Medio en Europa". En ARSUAGA, J. L. \& CARBONELL, E. (eds) Evolución humana en Europa y en los Yacimientos de la Sierra de Atapuerca.: 57-73.

- (1991): "Origene des grandes mamiferes pliopleistocenes d'Europe et migrations humaines". En BONIFAY, E. \& VANDERMERSCH B. (1991) Les premiers Européens editions du co- 
mité dels travaux historiques et scientifiques actes du 114 Congreés National des Societe savantes. 1989: 267-271.

VAUFREY, R. (1929): Les élephants nains des iles méditerranéennes et la question des isthmes Pléistocènes. Masson, èd., Paris, 1 vol. In $-4 .^{\circ}, 220 \mathrm{pp}$.

VEROSUB K. \& TCHERNOV E. (1991): “Résultats préliminaires de l'etude magnétostratigraphique d'une séquence sédimentaire a industrie humaine en Israel". In Bonifay \& Vandersmerch (1991), Les premiers européens Paris CTHS: 237-242.

Zazo C.; Goy, J. L; Hillaire-Marchal, C.; Dubrio C. L.; Hovos, M.; lorio, J.; Bardoli, T.; SomozA, L. \& SiLVA, P. G. (1994): "Variaciones en el nivel del mar. Estudios isotópicos 7, 5, y 1 en las costas peninsulares ( $S$ y $S E$ ) $e$ insulares españolas". En Gibraltar during the Quaternary, Gibraltar Museun INQUA Monografias 2: 26-35. 